\title{
Electrical and mechanical characterization of a flexible conducting composite
}

\author{
W. D. S. Deniz ${ }^{1}$ - E. A. Sousa ${ }^{1}$ - E. P. S. Arlindo ${ }^{1}$. \\ W. K. Sakamoto ${ }^{2}$ G. C. Fuzari Jr. ${ }^{1}$
}

Received: 4 August 2014/Revised: 26 March 2015/Accepted: 31 March 2015/

Published online: 10 April 2015

(C) Springer-Verlag Berlin Heidelberg 2015

\begin{abstract}
Composite films made of poly(vinylidene fluoride) (PVDF) and conducting particles of carbon black (CB) were prepared using a hot press. Using different volume fractions of $\mathrm{CB}$ filler, electrical properties of the samples were analyzed with current-voltage $(\mathrm{I} \times \mathrm{V})$ measurements and impedance spectroscopy. To help the discussion, percolation theory and simulation circuits based on Colie diagrams were used. The percolation threshold was found at $3 \mathrm{vol} \%$ of CB. At these volume fractions of filler, scanning electron microscopy (SEM) images showed connected particles in the polymer matrix, while in the higher volume content of filler, the particles started to form clusters. Furthermore, it was observed that increasing volume fractions up to 3 vol\%, the Young's modulus and the tensile strength of the composite film were higher than that of the pure polymer, although the rupture strain was decreased. Composite films with 3 vol\% of CB showed optimized electrical and mechanical properties and may be useful as an electrostatic dissipater.
\end{abstract}

Keywords Conductivity $\cdot$ Mechanical properties $\cdot$ Composite $\cdot$ Carbon black

G. C. Fuzari Jr.

gcfjunior@yahoo.com.br

1 Instituto de Ciências Exatas e da Terra, Universidade Federal de Mato Grosso, CEP 78600-00, Barra do Garças, MT, Brazil

2 Departamento de Física e Química, Universidade Estadual Paulista, CEP 15385-000, Ilha Solteira, SP, Brazil 


\section{Introduction}

In general, polymeric materials are flexible and have mechanical resistance, but they have low electrical conductivity. However, for some specific applications, a flexible material with non-negligible electrical conductivity is needed, as in the case of antistatic packaging $[1,2]$. To overcome these problems, conducting polymeric composites were fabricated, including semiconductor or conducting particles into the polymer matrix. The resulting material will combine with the mechanical properties and the process ability of the polymer with the electrical property of the conducting filler [1-7].

The composite sample can be obtained by melting the polymer matrix and incorporating the filler or including the filler during the polymerization of the matrix [8]. The properties of the composite depend on the nature of the inclusions and the matrix $[9,10]$. An interesting study is the percolation threshold, which indicated the limit of particle/matrix fraction rates where the insulating material became a conductor, i.e. the distribution of the particles in the matrix allowed at least one electrical flux path $[6,11-13]$.

In the present work, composite films were obtained by hot pressing the mixture of PVDF in powder form with carbon black (CB) particles. The decision to use these materials was made because PVDF is a thermoplastic polymer with good mechanical, optical and thermal properties and is an insulator [14]. On the other hand, $\mathrm{CB}$ has been used as conducting filler in polymeric composites, as dye inks, as catalyst in batteries and fuel cells, and as an additive to enhance the mechanical resistance of composites $[1,4,5,7]$. The main contribution of the present work on flexible conducting composites of ease processing is the correlation between the electrical property of the composite, studied by equivalent circuit, and its mechanical properties to propose a better composition to be used as an electrostatic dissipater.

\section{Experimental}

\section{Material}

PVDF $\alpha$-phase in powder form (SOLEF 1008/1001) with $1.78 \mathrm{~g} / \mathrm{cm}^{3}$ density was provided by Solvay. CB particles with $50 \mathrm{~nm}$ diameter, $1000 \mathrm{~m}^{2} / \mathrm{g}$ of area and density equal to $1.80 \mathrm{~g} / \mathrm{cm}^{3}$ were purchased from Union Carbide.

\section{Composite preparation}

CB particles were mixed with PVDF powder in mortar and macerated. Several particles/polymer volume fractions were used, such as 0, 0.5, 1.0, 3.0, 5.0 and 7.0. Each mixture was placed between two polyamide films (Kapton) and pressed at $185^{\circ} \mathrm{C}$ for $2 \mathrm{~min}$ with $7.0 \mathrm{MPa}$, using a temperature-controlled hydraulic press (Marconi model MA 098/A). After pressing, the composite film was quickly 
immersed in ice water for quenching. Aluminum electrodes were evaporated onto both sides of the composite film for electrical characterization.

\section{Characterization}

Conductivity measurements along the surface of the composite film and along its thickness direction were carried out using a current-voltage source (Keithley model 2657A). Field emission electron microscopy (FEG-SEM) was realized using a JEOL model 7500F at Institute of Chemistry, UNESP/Araraquara, and, for the impedance spectroscopy, a Multi Autolab PGSTAT 101 Metrohm with electrochemical impedance modulus FRA2 was used. The experimental data were adjusted using the EIS Spectrum Analyzer Software. The stress tests were carried out according to ASTM D 1708-10 standard, using a Universal test machine model WDW-300E with $12.5 \mathrm{~mm} / \mathrm{min}$ speed and $150 \mathrm{kN}$ loading cell.

\section{Results and discussion}

\section{Electrical conductivity}

The enhancement of DC conductivity of the composite samples with different volume fraction rates of $\mathrm{CB}$ was analyzed along the surface and thickness directions. In the surface measurements, there was a considerable increase of the conductivity from 3 vol\% of $\mathrm{CB}$, as shown in Fig. 1, indicating that between 1 and

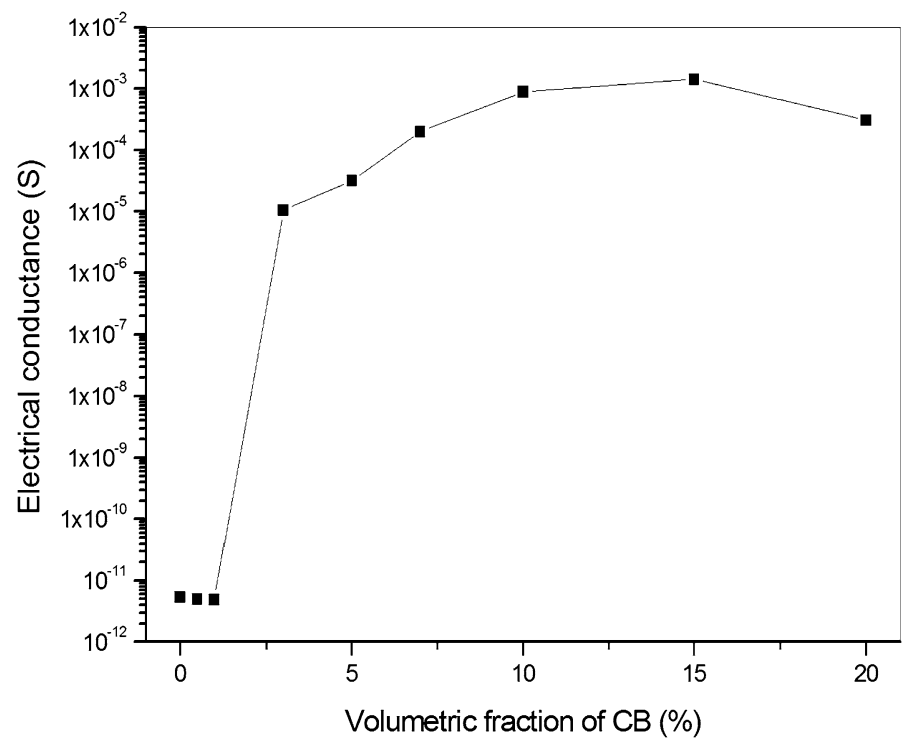

Fig. 1 Electrical conductance DC along the surface of PVDF/CB composites as a function of volumetric fraction of $\mathrm{CB}$ particles 
$3 \mathrm{vol} \%$ the material was no longer an insulator and started to be a conducting material. The behavior of the electrical conduction observed in the PVDF/CB composite was in agreement with the results obtained by other authors [15-17].

In this range of $\mathrm{CB}$ content, the conductance increased about 5 orders of magnitude, indicating an increasing conductivity of the material with the increase of conducting filler until the percolation threshold. The small increase of the conductivity after the critical concentration for percolation may be attributed to the better contact between the continuous electrical paths, since a small potential barrier allows charge conduction by hopping [7] or tunneling [15], or it may have been due to the enhancement of conducting channels until reaching the saturation when the localized thermal effects can hinder electrical conduction.

The result obtained along the thickness direction was similar, as shown in Fig. 2. The percolation threshold was also at $3 \mathrm{vol} \%$, indicating an isotropic material regarding the $\mathrm{CB}$ distribution into the PVDF matrix.

\section{FEG-SEM microscopy}

Figure 3a shows CB particles forming small clusters separated by the PVDF matrix. In a better view, in Fig. 3b-d, amplified from Fig. 3a, CB particles can be seen very close to each other through the insulator matrix, starting to form the electrical flux path. The analogous result was observed by Li et al. [18].

The micrograph shown in Fig. $4 \mathrm{e}, \mathrm{f}$ is related to the 3 vol\% of $\mathrm{CB}$ into the matrix. The increasing CB content, as expected, made the clusters closer to each other, creating an electrical flux path in the matrix. Consequently, the electrical

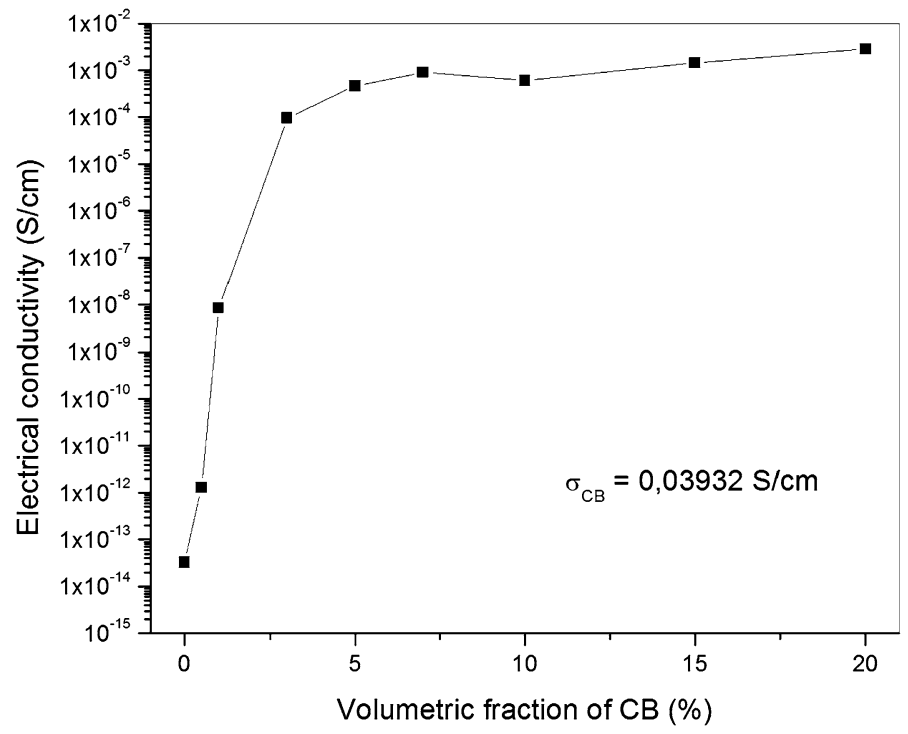

Fig. 2 Electrical conductivity DC along the thickness direction of PVDF/CB composites as a function of volumetric fraction of $\mathrm{CB}$ particles 

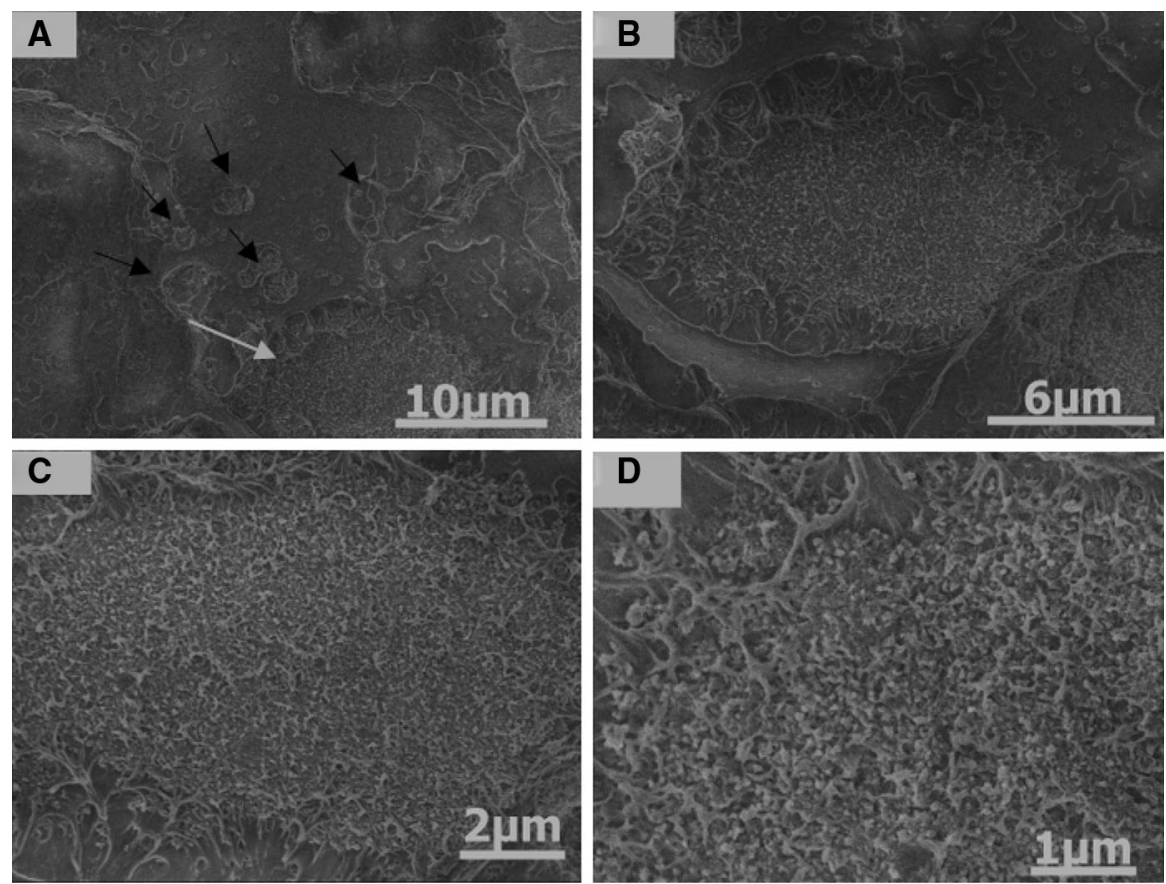

Fig. 3 FEG-SEM images of composite with 1 vol\% of CB content. The arrows show aggregates. The large arrow (in gray) indicates the aggregate that has been amplified
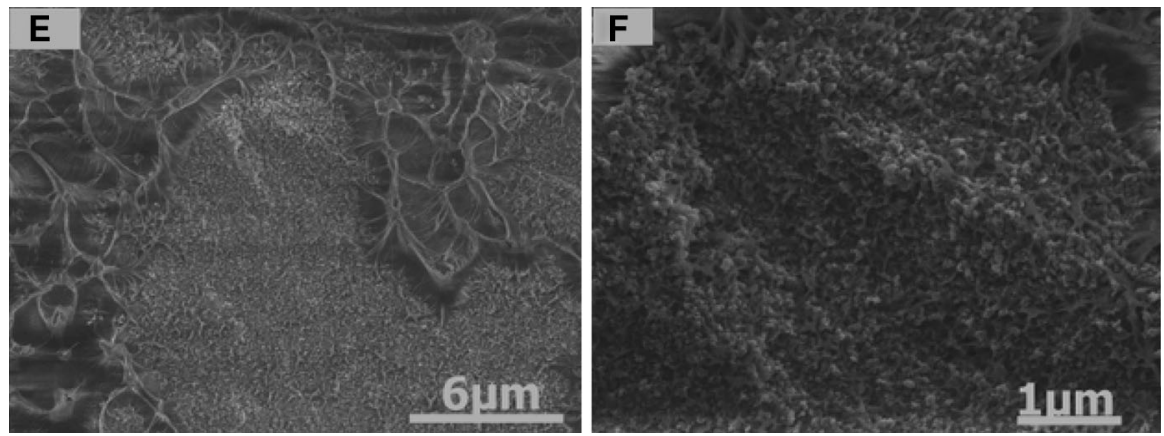

Fig. 4 FEG-SEM images of composite with 3 vol\% of CB content

conductivity was increased because the electron motion became easier. This means the percolation was reached.

For higher $\mathrm{CB}$ content, the particles' aggregate dimensions were more than $50 \mu \mathrm{m}$, and in this case, the mechanical properties of the film could be reduced (Fig. 5). 


\section{Impedance spectroscopy}

The use of equivalent circuits was quite common in the study of impedance behavior of a sample. This was a simple way to analyze electrical properties of a material, which can be understood as capacitors and resistors associated in different ways to explain the charge conduction and storage behavior [19, 20].

According to simulations, the equivalent circuit, which represented the electrical behavior of a percolated composite, was composed by two resistors and one capacitor associated, as shown in Fig. 6. $R_{1}$ was the contact resistance (interface electrode sample) and was used to measure the impedance. $R_{2}$ represented the conductive phase $(\mathrm{CB})$, and $C_{1}$, in parallel, represented the capacitive effect of the polymer matrix. There were two distinct behaviors for the electrical charge: it should be storage in the matrix (interface charge), or it would flow through the conducting path as effective electrical current.

For composites with 7 vol\% of $\mathrm{CB}$, the adjustment was perfect, as shown in Fig. 7d. However, for 3 vol\% (Fig. 7a) and 5 vol\% (Fig. 7b), the capacitor needed
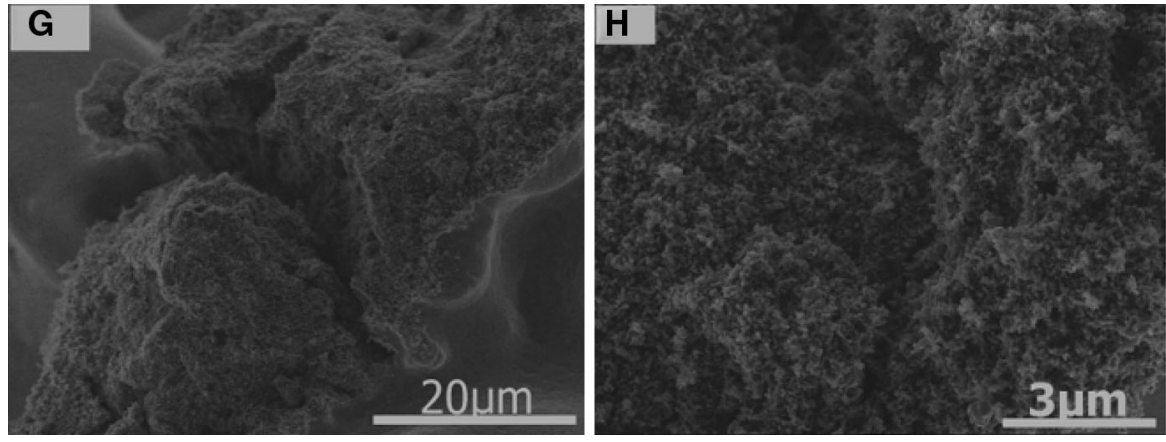

Fig. 5 FEG-SEM images of composite with 5 vol\% of CB content

Fig. 6 Illustration of the equivalent electric circuit behavior of the percolated composite films (7 vol\% of $\mathrm{CB})$

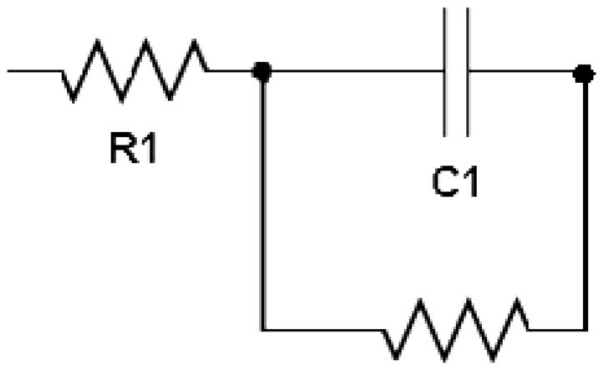

R2

Fig. 7 Simulation using equivalent circuits - composites above the percolation threshold. Composites with a 3 vol\%, b $5 \mathrm{vol} \%$, c 7 vol\% (simulated CPE) and d 7 vol\% (simulated with capacitor) of CB content 

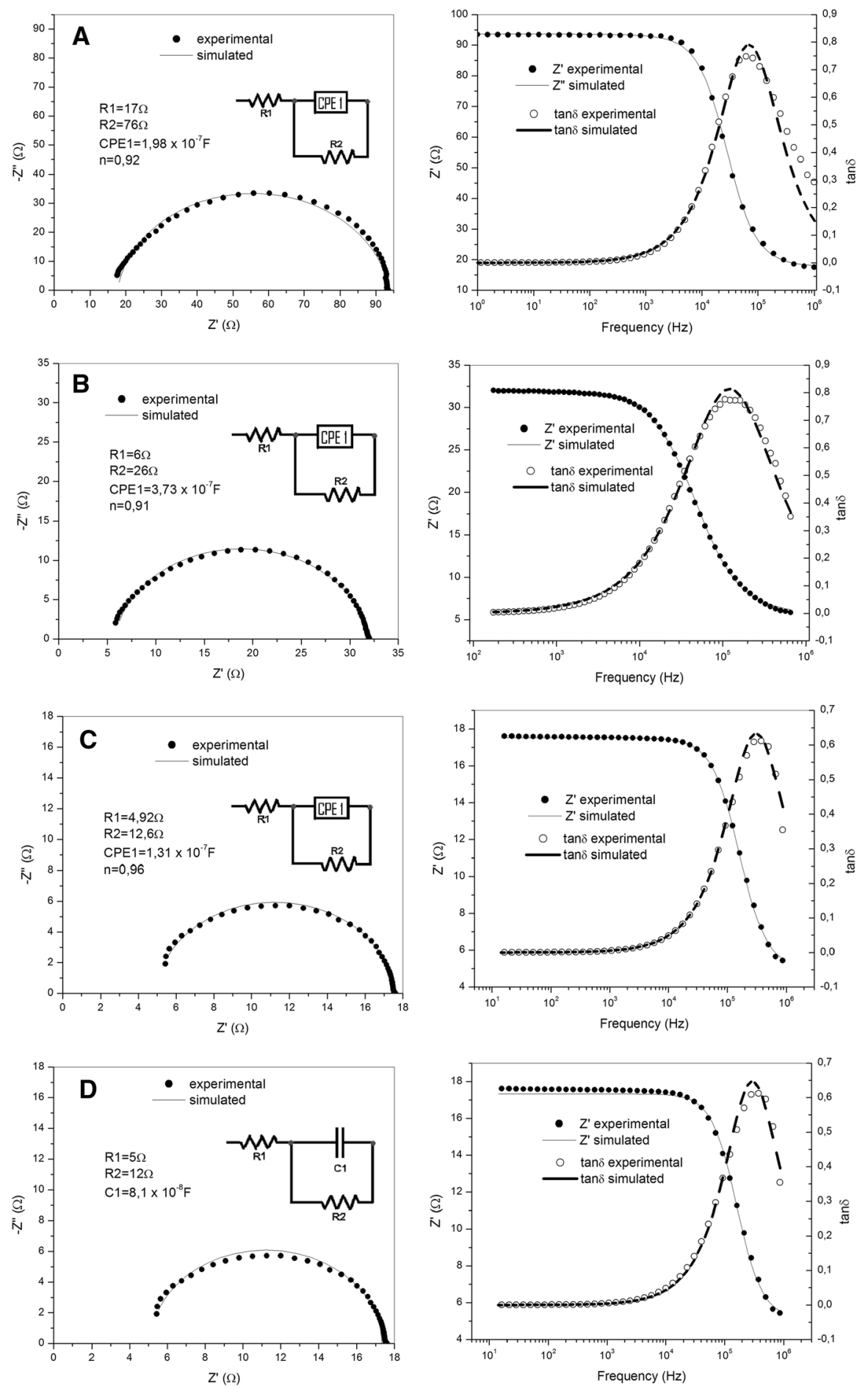

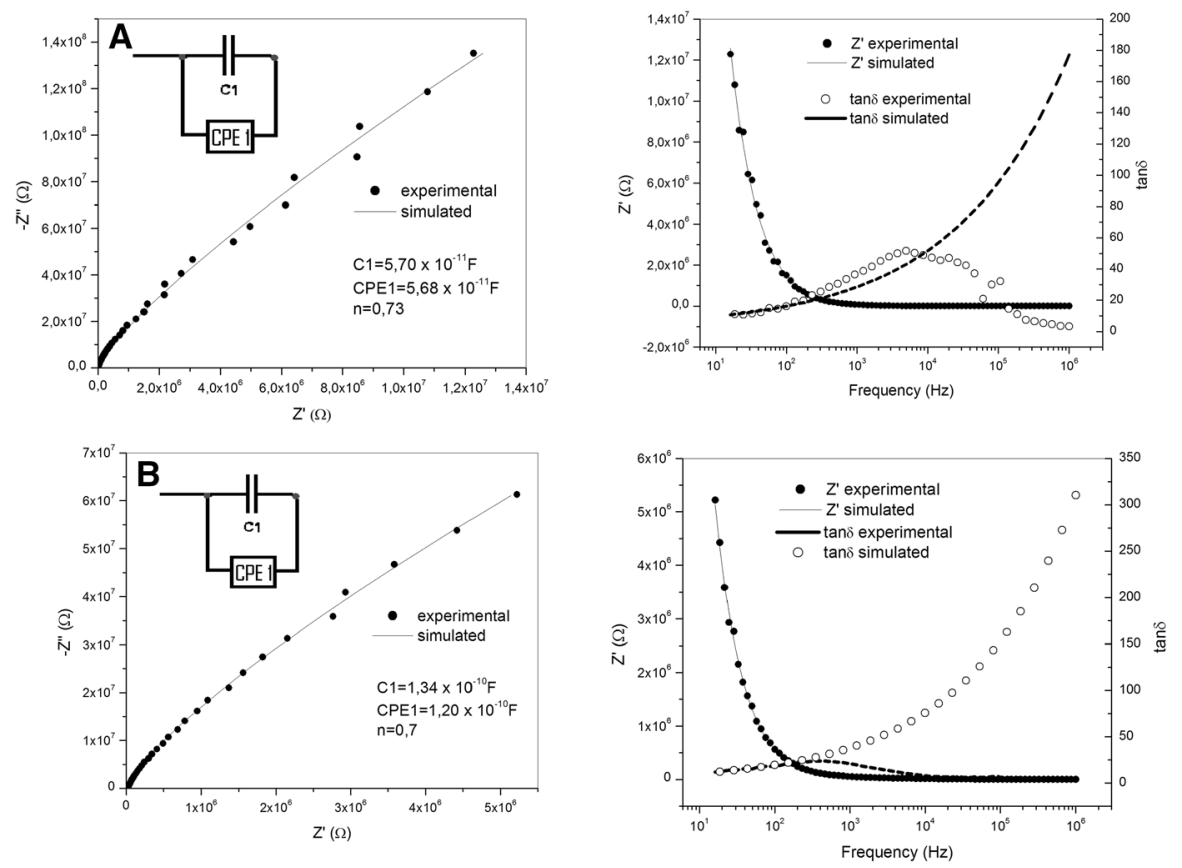

Fig. 8 Simulation with equivalent circuits—composites below the percolation threshold: a pure PVDF and b PVDF with 1 vol\% of CB content

to be changed for a constant phase element (CPE). The CPE has the value of $n$, near to unit, indicating that it was approximately a charge storage (capacitor) device. It was possible to note that CPE became a perfect capacitor as the CB content increased. In the simulation of the equivalent circuit for a composite with $7 \mathrm{vol} \%$, the value of $n$ was equal to 0.96 , and the CPE could be substituted for a capacitor and resistor (Fig. 7d).

For PVDF films or composites with a CB concentration lower than the percolation threshold, the results were similar. The adjustments became harder, and the equivalent circuit was like a capacitor associated, in parallel, with a CPE. In this case there was no conduction, and the charge was essentially storage. As shown in Fig. 8a, b, the adjustment of dielectric loss was very hard. In these cases, the use of Lock-In amplifier and current discharge techniques was better [21].

The electrode diameter, and film thickness, of the samples is shown in the Table 1 . These values, together with the adjusted $R_{2}$ value, gave the conductivity $\sigma$ of the composite, using Eq. (1).

$$
\sigma=\frac{i A}{V l}
$$

where $i$ is the electrical current intensity, $V$ is the applied voltage, $A$ is the electrode area and $l$ is the sample thickness. 
Table 1 Diameter and thickness of the films obtained

\begin{tabular}{lcc}
\hline Sample & Thickness $(\mu \mathrm{m})$ & Diameter of electrode $(\mathrm{mm})$ \\
\hline PVDF & 100 & 8.5 \\
PVDF/CB 1 vol\% & 70 & 10.0 \\
PVDF/CB 3 vol\% & 90 & 8.5 \\
PVDF/CB 5 vol\% & 120 & 8.5 \\
PVDF/CB 7 vol\% & 270 & 8.5 \\
\hline
\end{tabular}

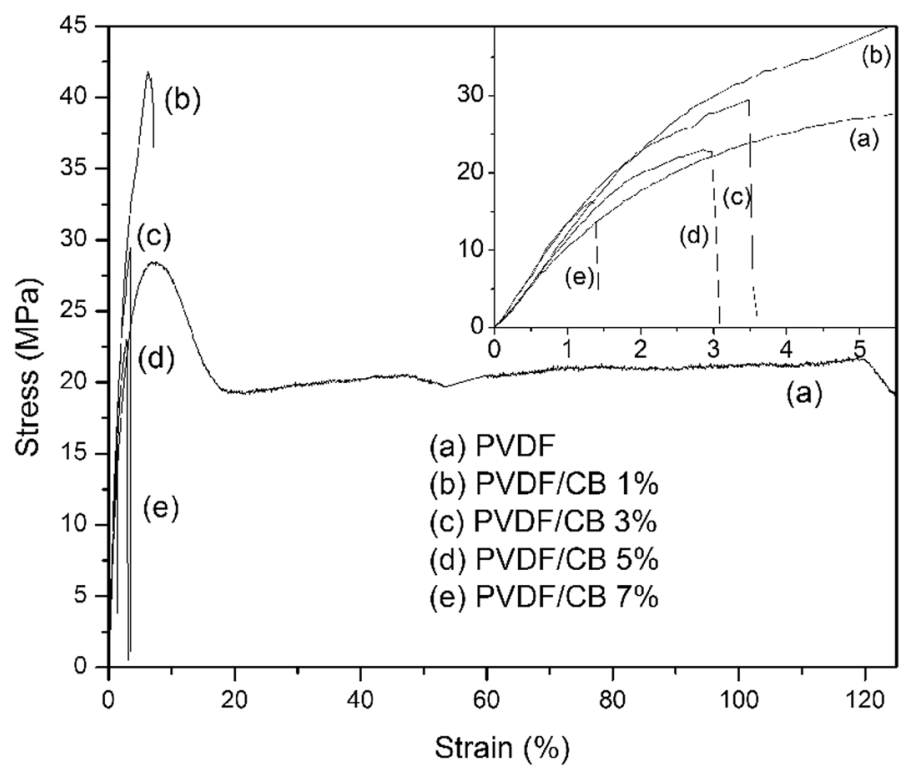

Fig. 9 Stress-strain tests

The values of $\sigma$ obtained by Eq. (1) were in agreement with the values obtained by $I \times V$ measurement. For example, the conductivity was around $1 \times 10^{-4} \mathrm{~S} / \mathrm{cm}$ for the composite with $3 \mathrm{vol} \%$.

\section{Stress-strain tests}

Figure 9 shows the results of the stress-strain tests of PVDF/CB composites in the range of $0-7 \mathrm{vol} \%$ of $\mathrm{CB}$. In general, the rupture strain of composites decreased as the $\mathrm{CB}$ content was increased. For composites with 1 and $3 \mathrm{vol} \%$, the tensile strength was higher than the pure PVDF, but for 5 and 7 vol\%, this value was lower when compared with PVDF; indicating that until 3 vol\%, the CB was a reinforcing filler since it must restrict the flow of the chains by localized interactions [8, 10, 18].

Table 2 shows the mechanical parameters calculated from Fig. 9. When the CB content was high, the coupling of large clusters of $\mathrm{CB}$ restricted its mechanical 
Table 2 Tensile properties of films

\begin{tabular}{llcllc}
\hline $\begin{array}{l}\text { Volumetric fraction } \\
\text { of CB (\%) }\end{array}$ & $\begin{array}{l}\text { Young's } \\
\text { modulus (GPa) }\end{array}$ & $\begin{array}{l}\text { Rupture } \\
\text { strain (\%) }\end{array}$ & $\begin{array}{l}\text { Rupture } \\
\text { stress (MPa) }\end{array}$ & $\begin{array}{l}\text { Limit of tensile } \\
\text { strength (MPa) }\end{array}$ & $\begin{array}{l}\text { Yield } \\
\text { strength } \\
\text { (Mpa) }\end{array}$ \\
\hline 0 & 1.06 & 120.0 & 21.6 & 28.3 & 7.2 \\
1 & 1.21 & 7.0 & 40.0 & 42.0 & 14.0 \\
3 & 1.46 & 3.5 & 30.0 & 30.0 & 13.0 \\
5 & 1.18 & 3.0 & 23.0 & 23.0 & 11.0 \\
7 & 1.40 & 1.4 & 16.0 & 16.0 & 5.2 \\
\hline
\end{tabular}

strength. Although the Young's modulus of composite with 7 vol\% was higher than the $5 \mathrm{vol} \%$, the other parameters were lower, and the sample was more fragile. The formation of neck during the deformation test was observed only in pure PVDF.

\section{Conclusions}

PVDF/CB films with different CB contents were obtained by mixing the powder of both components with subsequent hot pressing. The electrical conductivity of the new material increased as the CB content was increased, allowing the determination of the percolation threshold of the composite samples, which occurred around 3 vol\% of filler.

The composite was isotropic, in regard to the electrical property, since the percolation threshold appeared at the same volume fraction of filler in both directions of measurement. Pure PVDF film and composite under percolation threshold behaved as a capacitor, restricting the current flow and working as a charge storage device. Over the percolation, there are two possibilities: there may be an accumulation of charges on the polymer interface, or the charges could flow along the conducting channels (percolated CB phase).

Mechanical properties of the composite could be enhanced using low volume fraction rates of $\mathrm{CB}$. The value of $3 \mathrm{vol} \%$ appeared as a better composition, and the composite showed a potential application as an electrostatic dissipater.

Acknowledgments Thank you to Fundação de Amparo à Pesquisa do Estado de Mato Grosso (FAPEMAT) for the financial support. WKS also thanks Fundação de Amparo à Pesquisa do Estado de São Paulo (FAPESP) (CEPID 2013/07296-2 and INCTMN 2008/57872-1) and Conselho Nacional de Desenvolvimento Científico e Tecnológico (CNPq) (INCTMN 573636/2008-7).

\section{References}

1. Aal NA, El-Tantawy F, Al-Hajry A, Bououdina M (2007) New antistatic charge and electromagnetic shielding effectiveness from conductive epoxy resin/plasticized carbon black composites. Polym Compos 29(2):125-132

2. Strümpler R, Glatz-Reichenbach J (1999) Conducting polymer composites. J Electroceram 3(4):329-346 
3. Arlindo EPS, Lucindo JA, Bastos CMO, Emmel PD, Orlandi MO (2012) Electrical and optical properties of conductive and transparent ITO@PMMA. Nanocompos J Phys Chem C 116(23): 12946-12952

4. Liang J, Yang Q (2007) Aggregate structure and percolation behavior in polymer/carbon Black conductive composites. J Appl Phys NY 102:083508.1-083508.5

5. Li F et al (2000) Polyurethane/conducting carbon black composites: structure, electric conductivity, strain recovery behavior, and their relationships. J Appl Polym Sci NY 75:68-77

6. Bunde A, Dieterich W (2000) Percolation in composites. J Electroceram Dordr 5(2):81-92

7. Da Silva MJ, Kanda DHF, Nagashima HN (2012) Mechanism of charge transport in castor oil-based polyurethane/carbon black composite (PU/CB). J Noncryst Solids 358:270-275

8. Calister WD, Rethwisch DG (2010) Materials science and engineering, 8th edn. Wiley, New York

9. Jing X, Zhao W (2000) The effect of particle size on electric conducting percolation threshold in polymer/conducting particle composites. J Mater Sci Lett Lond 9:377-379

10. Fu S-Y, Feng X-Q, Lauke B, Mai Y-W (2008) Effects of particle size, particle/matrix interface adhesion and particle loading on mechanical properties of particulate-polymer composites. Compos Part B 39:933-961

11. Berkowitz B, Ewing RP (1998) Percolation theory and network modeling applications in soil physics. Surv Geophys Dordr 19(1):23-72

12. Yang QQ, Liang JZ (2008) A percolation model for insulator-metal transition in polymer-conductor composites. Appl Phys Lett NY 93:131918.1-131918.3

13. Derrida B, Zabolitzky JG, Vannimenus J, Stauffer DJ (1984) A transfer matrix program to calculate the conductivity of random resistor networks. J Stat Phys 36:31

14. Lovinger AJ (1982) Poly(Vinylidene Fluoride). In: Basset DC (ed) Developments in crystalline polymers-1, vol 33. Springer, Netherlands, pp 195-273

15. Balberg I (2001) A comprehensive picture of the electrical phenomena in carbon black-polymer composites. Carbon 40:139-143

16. Probst N, Grivei E (2002) Structure and electrical properties of carbon black. Carbon 40:201-205

17. Zhao Z, Yu W, He X, Chen X (2003) The conduction mechanism of carbon black filled poly(vinylidene fluoride) composite. Mater Lett 57:3082-3088

18. Li F, Qi L, Yang J, Xu M, Luo X, Ma D (1999) Polyurethane/conducting carbon black composites: structure, electric conductivity, strain recovery behavior, and their relationships. J Appl Polym Sci 75(1):68-77

19. Jonscher AK (1977) The universal dielectric response. Nature Lond 267(5613):673-679

20. Cole RH (1965) Relaxation processes in dielectrics. J Cell Comp Physiol NY 66(3):13-25

21. Hamon BV (1952) An approximate method for deducing dielectric loss factor from direct-current measurements. Proc IEEE Part IV Monograph 27:151-155 